\title{
le projet communautaire COSA : un exemple d'intercomparaison de codes de calcul géomécaniques pour le sel
}

\author{
the community project COSA : \\ an example of intercomparison exercise \\ for rock mechanics computer codes for salt
}

\author{
B. CÔME \\ Commission des Communautés Européennes*
}

\section{Résumé}

Dans le cadre du programme communautaire "Gestion et Stockage des Déchets Radioactifs", on a réalisé un exercice d'intercomparaison de codes de calcul géomécaniques pour le sel (COSA), associant dix équipes des paysmembres, la firme britannique ATKINS agissant comme secrétariat technique. Cet exercice avait pour but :

- de tester l'efficacité et la facilité d'utilisation des codes de calcul sur ordinateur examinés:

- d'évaluer leur précision numérique:

- de vérifier leur aptitude à reproduire correctement la réalité physique.

L'article présente les principaux enseignements tirés de ce projet, particulièrement en ce qui concerne le troisième objectif, encore appelé "validation" des codes de calcul.

\section{Abstract}

In the framework of the Community programme on "Management and Storage of Radioactive Waste", an intercomparison exercise for rock-mechanics computer codes for salt (COSA) was carried out. It put together the efforts of 10 teams of Member States, with the British firm ATKINS acting as technical secretariat.

The aims of the exercise were as follows:

- testing efficiency and user-friendliness of the participating computer codes;

- evaluating the numerical accuracy of the codes:

- verifying their aptitude to correctly replicate real life phenomena.

This paper describes the findings of this project, mainly concerning the third objective, the so-called "validation" of computer codes.

\footnotetext{
- Direction Générale de la Science, de la Recherche et du Développement, rue de la Loi, 200. B. 1049 Bruxelles.
} 


\section{INTRODUCTION}

Parmi les études relatives à l'évacuation géologique des déchets radioactifs, le calcul des contraintes mécaniques dans le massif-hôte occupe une place importante. Cette investigation est en effet nécessaire à deux niveaux :

- d'abord, pour concevoir et construire des dépôts souterrains profonds stables pendant la phase d'exploitation, c'est-à-dire la mise en place proprement dite des déchets;

- ensuite, pour prédire le comportement mécanique d'ensemble du massif-hôte après la fermeture du dépôt, par exemple sous l'effet du dégagement thermique de déchets de haute-activité

Compte tenu de la complexité des géométries à envisager et du comportement rhéologique des matériaux géologiques, ce genre de calcul est réalisé par des codes sur ordinateur utilisant le plus souvent la méthode des éléments finis.

Depuis une dizaine d'années, la Commission des Communautés Européennes participe à la mise au point de tels outils de calculs, dans le cadre de son programme de R \& D sur * la gestion et le stockage des déchets radioactifs » (SIMON, ed., 1985). En novembre 1984, les principales équipes européennes compétentes dans ce domaine furent invitées à participer au projet COSA (Comparison of Computer Codes for Salt), visant à mesurer les capacités des outils de calcul ainsi mis au point. Le choix du sel comme matériau candidat résultait de la vaste expérience déjà acquise à son propos dans le cadre du programme communautaire.

Une première phase du projet COSA s'est achevée en juillet 1986 (LOWE, 1986). Étant le premier de son genre à l'échelle communautaire, cet exercice volontairement limité s'était donné comme objectifs :

- de tester l'efficacité et la facilité d'emploi des codes de calcul considérés:

- d'évaluer la précision numérique des codes étudiés;

- de vérifier leur aptitude à reproduire correctement la réalité physique.

Plus qualitativement, on a créé par ce biais un courant d'échanges accrus entre les diverses équipes; également, la procédure employée pour cet exercice a été testée en vue d'améliorations ultérieures éventuelles.

De tels projets ont déjà été réalisés hors de la Communauté, en particulier aux U.S.A. dans le cadre du «WIPP Benchmark * organisé par le laboratoire SANDIA (MORGAN, 1981). Dans le cadre de COSA, on a cherché à éviter toute duplication avec de tels prédécesseurs; bien évidemment, on a tenu compte des enseignements ainsi acquis.

\section{ORGANISATION DE L'EXERCICE}

\subsection{Participants}

Dix équipes européennes ont participé au projet COSA, la firme britannique d'ingéniérie ATKINS R. \& D. à Epsom, agissant comme secrétaire scientifique et coordinateur impartial pour le compte de la Commission. Ce sont :

\section{En Belgique :}

La firme FORAKY, associée au Centre d'Études de l'Énergie Nucléaire (C.E.N./S.C.K.) et au Laboratoire du Génie Civil de l'université de Louvain-la-Neuve (L.G.C.) :

En République Fédérale d'Allemagne :

La Rheinisch-Westfälische Technische Hochschule (R.W.T.H.), Aix-la-Chapelle; La Gesellschaft für Strahlen- und Umweltforschung (G.S.F.), Braunschweig; Le Kernforschungszentrum Karlsruhe (KfK), Karisruhe;

Au Danemark :

Le Département d'Ingéniérie du RIS $\emptyset$ Laboratory (RIS $\emptyset)$, Roskilde ;

En France :

Le Laboratoire de Mécanique des Solides (L.M.S.) de l'École Polytechnique, Palaiseau :

Le Centre de Mécanique des Roches de l'École des Mines (E.M.P.), Fontainebleau;

Le Laboratoire d'Analyse Mécanique des Structures, Département des Études Mécaniques et Thermiques, du Commissariat à l'Énergie Atomique, Saclay (C.E.A.-D.E.M.T.)

En Italie :

L'Istituto Sperimentale Modelli e Strutture (I.S.M.E.S.), Bergame.

Aux Pays-Bas :

L'Energieonderzoek Centrum Nederlands (E.C.N.), Petten.

\subsection{Codes de calcul testés}

Les codes utilisés pour l'exercice sont listés dans le tableau 1 ; ceux figurant entre parenthèses sont destinés spécifiquement aux calculs thermiques.

\subsection{Types de problèmes}

L'excercice a été divisé en deux «niveaux" (ou «benchmarks $x$ ) correspondant aux principaux objectifs :

\section{Niveau 1}

Problème théorique simple destiné à tester les aspects numériques des codes, tels que la stabilité et la précision des solutions obtenues.

\section{Niveau 2}

Re-calcul, par les participants, d'un essai de laboratoire représentatif de conditions d'évacuation de déchets radioactifs dans le sel. Les résultats des calculs ont donc été comparés non seulement entre eux, mais aussi avec les mesures expérimentales.

En parallèle à ces aspects techniques, des paramètres plus qualitatifs tels que l'efficacité des codes, et leur facilité d'emploi, ont été examinés. Pour ceci, les participants ont d'une part utilisé un petit «programmeétalon " commun à tous; et d'autre part, un représen- 
tant d'ATKINS a assisté aux calculs du Niveau 1 en compagnie des spécialistes concernés.

On notera que, pour des raisons internes indépendantes de l'exercice, le laboratoire RIS $\varnothing$ (D.K.) s'était retiré du projet après l'achèvement du Niveau 1 et n'a donc pas participé au Niveau 2.

Les paragraphes qui suivent vont se concentrer sur les enseignements du a Niveau 2 »comme étant celui lié le plus intimement au délicat problème de la * validation * des outils de calcul numérique.

\section{PROBLÈME ET RÉSULTATS DU «NIVEAU 2» DU PROJET COSA}

\subsection{Description du cas expérimental}

L'essai - dénommé R.T.A - a été réalisé à l'Université de Delft (N.L.), département *exploitation minière». C'est le quatrième d'une série d'essais réalisés à l'origine dans le cadre d'un contrat de recherche communautaire (ROEST, 1985).

Un cube de sel d'arête $300 \mathrm{~mm}$ et pourvu en son centre d'un forage axial de diamètre $66 \mathrm{~mm}$ est comprimé dans les trois directions par les plateaux rigides d'une presse ; ensuite, un * réchaud " électrique est introduit dans le forage et provoque une élévation de tempéra-

Tableau I. - Participants et codes utilisés.

\begin{tabular}{|c|c|}
\hline FIRMES & CODES \\
\hline $\begin{array}{c}\text { FORAKY }+ \text { CEN/SCK } \\
+ \text { LGC } \\
\text { RWTH }\end{array}$ & CREEP \\
GSF & MAUS \\
(FAST-BEST) & ADINA/ANSALT \\
KFK & ADINAT/ANTEMP) \\
RIS $\emptyset$ & (ASYTE) \\
LMS & ADINA \\
EMP & ASTREA \\
(ASTHER) & CYSIPHE/VIPLEF \\
(CHEF) & INCA \\
CEA-DEMT & (DELFINE) \\
ISMES & GAMBLE \\
ECN & GOLIA/MARC/ANSYS \\
\hline
\end{tabular}

ture. La puissance thermique émise est connue, ainsi que les températures à des endroits choisis (via des thermocouples), et les déplacements de la paroi intérieure du forage et des plateaux de la presse. L'essai dure 13,5 heures. Le sel provient de l'horizon dit « $\mathrm{Na}_{2}$ " de la mine de Asse (R.F.A.) et a fait l'objet, depuis des années, d'investigations fouillées quant à sa rhéologie et à ses propriétés thermiques. Ces travaux ont été réalisés surtout à la G.S.F. et au Bundesanstalt für Geowissenschaften und Rohstoffe à Hanovre (R.F.A.).

Les historiques de chargement et d'échauffement étant donnés, ainsi que les propriétés du sel, on a demandé de calculer

- les températures en certains points;

- la convergence intérieure du forage et les mouvements de certains points du sel;

- quelques évolutions de contraintes en des points spécifiés.

Les figures 1 et 2 représentent respectivement l'arrangement de la presse et celui du bloc de sel instrumenté. Les propriétés du sel $\mathrm{Na}_{2}$ sont décrites (éventuellement en fonction de la température) comme suit :

- Masse volumique : $2187 \mathrm{~kg} / \mathrm{m}^{3}$

- Conductivité thermique :

$\lambda=5,734-1,838.10^{-2} \theta+2.86 .10^{-5} \theta^{2}$

$-1,51 \cdot 10^{-8} \theta^{3}\left(\lambda\right.$ en $\mathrm{W} / \mathrm{m} /{ }^{\circ} \mathrm{C}, \theta$ en $\left.{ }^{\circ} \mathrm{C}\right)$

- Capacité calorifique :

$\mathrm{Cp}=1,8705 \cdot 10^{6}+3,8772 \cdot 10^{2} \theta\left(\mathrm{J} / \mathrm{m}^{3} /{ }^{\circ} \mathrm{C}\right)$

- Module d'Young : $\mathrm{E}=24 \mathrm{GPa}$

- Coefficient de Poisson : $\nu=0,27$

- Coefficient d'expansion thermique : $4,2 \cdot 10^{-5}{ }^{\circ} \mathrm{C}^{-1}$

Les autres propriétés mécaniques, et notamment la loi de fluage, sont discutées plus loin. Quant à l'acier des plateaux, on le décrit par ses propriétés thermiques et mécaniques indépendantes de la température :

- masse volumique : $7830 \mathrm{~kg} / \mathrm{m}^{3}$

- Conductivité thermique $: 60 \mathrm{~W} / \mathrm{m} /{ }^{\circ} \mathrm{C}$

- Capacité calorifique : $3,758.10^{6} \mathrm{~J} / \mathrm{m}^{3} /{ }^{\circ} \mathrm{C}$

- Module d'Young : $210 \mathrm{GPa}$

- Coefficient de Poisson : 0,3

\subsection{Calcul thermique}

En préalable au calcul des contraintes et déplacement, une première partie de l'exercice consiste à déterminer le champ des températures dans le sel. Pour cela, on a assimilé le cube à un cylindre de sel a inscrit », ce qui permet de calculer le problème en symétrie de révolution; de plus, on admet qu'il y a symétrie par rapport au plan médian perpendiculaire au forage. Les plateaux de la presse sont pris en compte respectivement comme des disques et une couronne circulaire de volume convenable. Le flux de chaleur dégagée par le réchaud est supposé appliqué sur toute la paroi du forage, comme suit :

\begin{tabular}{|l|c|c|c|c|c|c|}
\hline Temps (minutes) & 0 & 90 & 150 & 540 & 600 & 810 \\
\hline Flux $\left(\mathrm{W} / \mathrm{cm}^{2}\right)$ & 0 & 0 & 1,61 & 1,61 & 0 & 0 \\
\hline
\end{tabular}

Entre ces dates, le flux évolue linéairement avec le temps. Le flux maximal correspond à une puissance de $1 \mathrm{~kW}$ consommée par le réchaud. 


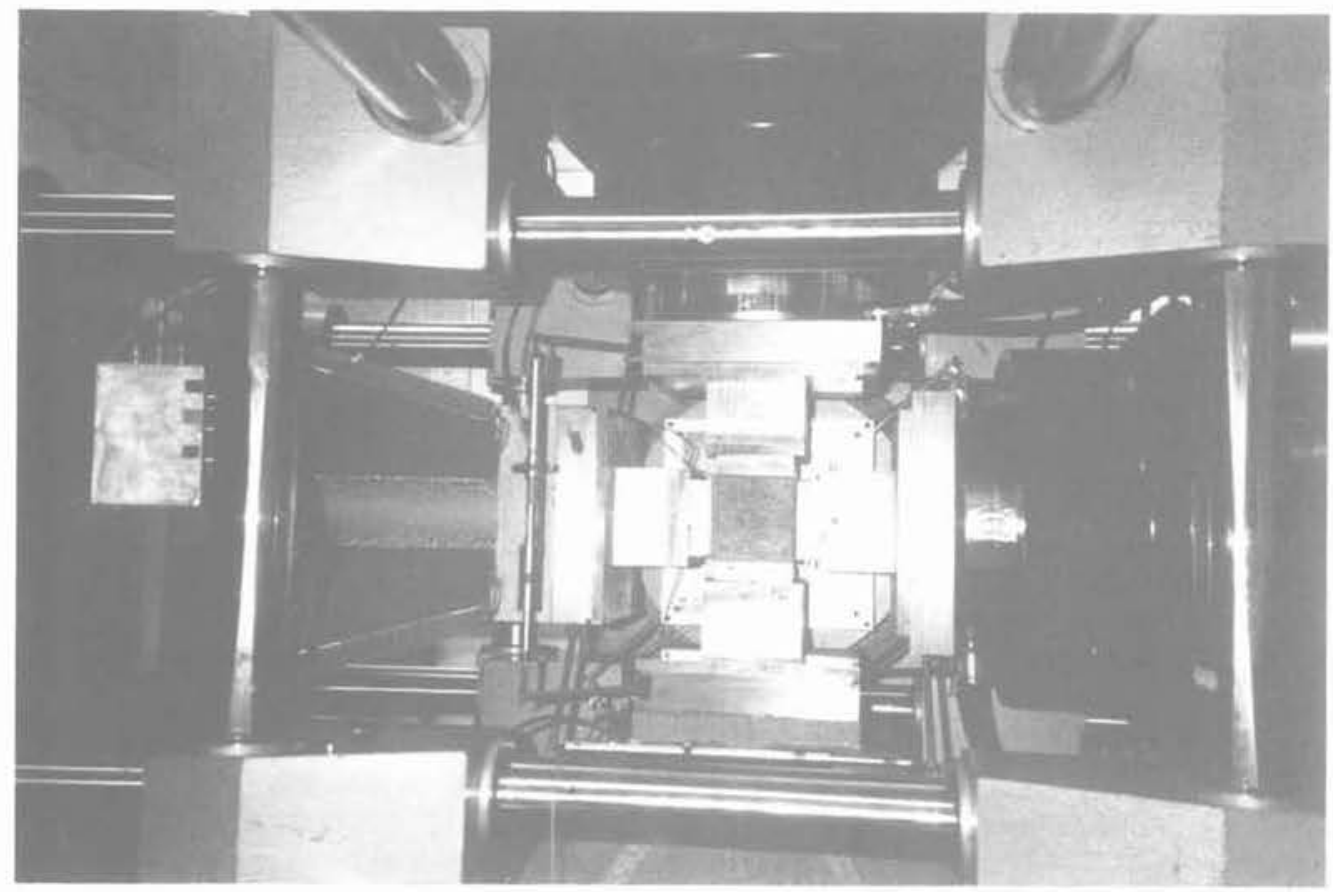

Fig. 1. - Vue de la presse triaxiale (le plateau antérieur est enlevé). Fig. 1. - View of the triaxial press (front platen removed).

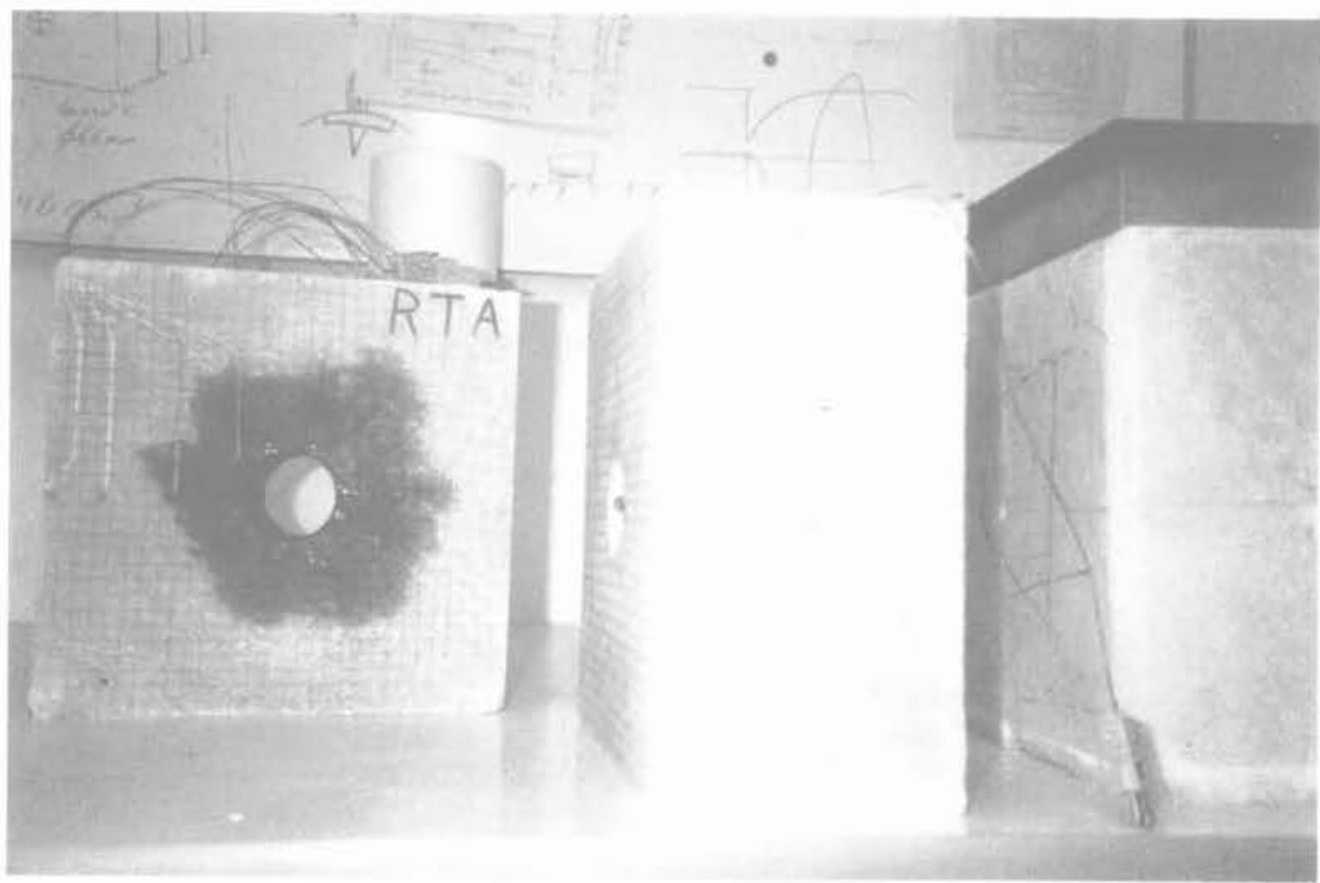

Fig. 2. - Le bloc de sel "RTA » (arête : environ $30 \mathrm{~cm}$ ).

Fig. 2. - The "RTA" salt block (edge is about $30 \mathrm{~cm}$ ). 


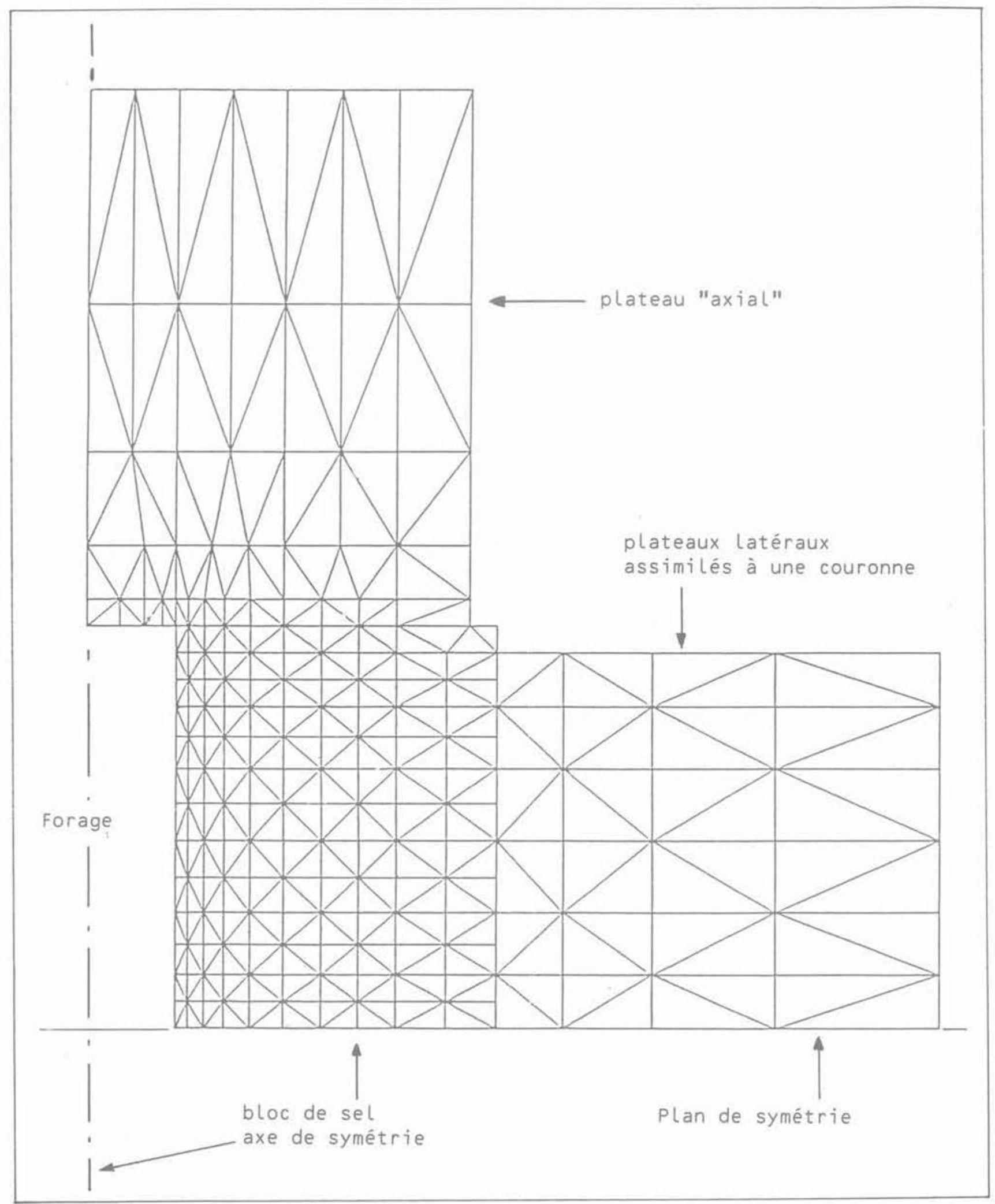

Fig. 3. - Exemple de maillage d'éléments finis utilisé pour le calcul thermique du "Niveau 2 ». Fig. 3. - Example of finite element mesh used for the "Benchmark 2" thermal calculation. 
Pour simplifier les calculs, les températures mesurées à la surface extérieure des plaques d'acier ont été prises comme conditions aux limites prescrites.

La figure 3 donne un exemple de maillage d'éléments finis utilisés pour ce calcul.

La figure 4 permet de comparer l'évolution de la température à la paroi du forage, respectivement mesurée et prédite par l'ensemble des codes. On ne peut que constater le remarquable accord entre les deux courbes, compte tenu des nombreuses simplifications nécessaires pour un calcul d'ampleur raisonnable.

\subsection{Calcul mécanique} (contraintes et déformations). Cas «de base».

De façon à mettre l'ensemble des participants sur un pied d'égalité, on a d'abord arrêté en commun les caractéristiques d'un cas \& de base * calculable par tous les codes, sur lequel des variations seraient possibles. Pour ce cas, on effectue le calcul d'une moitié du cube, assimilé à un cylindre, donc en symétrie de révolution. Seul le plateau d'acier exerçant le chargement axial est pris en compte; la surface latérale du bloc de sel est soumise directement à une pression imposée. Le chargement montre l'historique ci-dessous :

\begin{tabular}{|l|r|r|r|r|r|r|}
\hline Temps (minutes) & 0 & 30 & 60 & 750 & 780 & 810 \\
\hline $\begin{array}{l}\text { Forces appliquées } \\
(\mathrm{kN})\end{array}$ & 250 & 250 & 2500 & 2500 & 250 & 250 \\
\hline
\end{tabular}

L'évolution du chargement est linéaire entre ces dates. On admet qu'il n'y a pas de frottement entre le sel et lacier. A ce chargement externe, viennent se superposer les contraintes dues à l'échauffement.

Le problème le plus délicat concerne la rhéologie du matériau. Pour ce cas de base, la déformation plastique instantanée est négligée. D'autre part, compte tenu de la brièveté de l'essai, seul le fluage "primaire » est à considérer. Celui-ci est décrit par l'équation :

$$
\dot{\varepsilon}_{\text {eq }}^{c t}=m \cdot B \cdot \exp (-m \cdot t) \cdot \sigma_{e q}^{n} \cdot \exp \left(-Q_{1} / R T\right)
$$

(le fluage secondaire étant négligeable lorsque t est petit). Une simplification ultérieure consiste à prendre $\mathrm{mt} \simeq 0$, et à se ramener ainsi à un fluage «pseudopermanent ». En l'absence de données plus complètes, c'est cette approche qu'on a retenue pour le cas de base.

On a adopté :

$\mathrm{m}=0.35$ jour $^{-1} ; \mathrm{B}=0.21 ; \mathrm{n}=5$;

$Q_{1}=44,8,10^{3} \mathrm{~J}$

Une première série de résultats de calcul fut obtenue et comparée aux enregistrements expérimentaux; la figure 5 concerne ainsi le calcul et la mesure de la convergence radiale du forage au voisinage de son plan médian. Prise séparément, chacune des courbes pouvait paraître vraisemblable compte tenu des simplifications du modèle. Un examen plus attentif permit d'établir que la majorité des divergences entre les calculs s'expliquaient par des erreurs "humaines» au moment de l'entrée des données dans les codes sur ordinateur. Une nouvelle série de calculs fut donc réali-

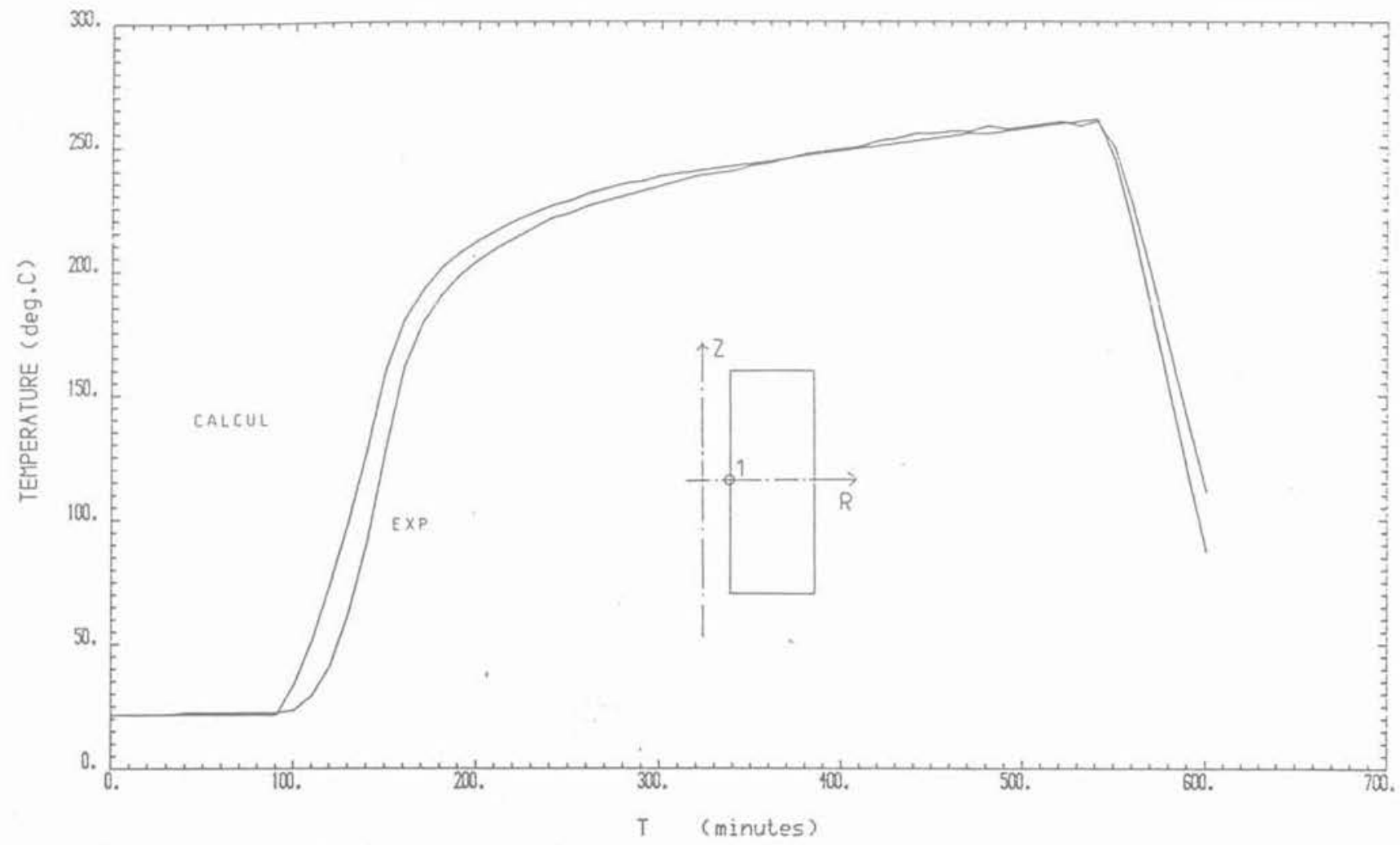

Fig. 4. - Niveau 2. Comparaison de la température mesurée et des températures calculées (tous codes confondus) sur la paroi du forage.

Fig. 4. - Benchmark 2. Comparison of measured and calculated (all codes) temperature evolution at borehole wall. 


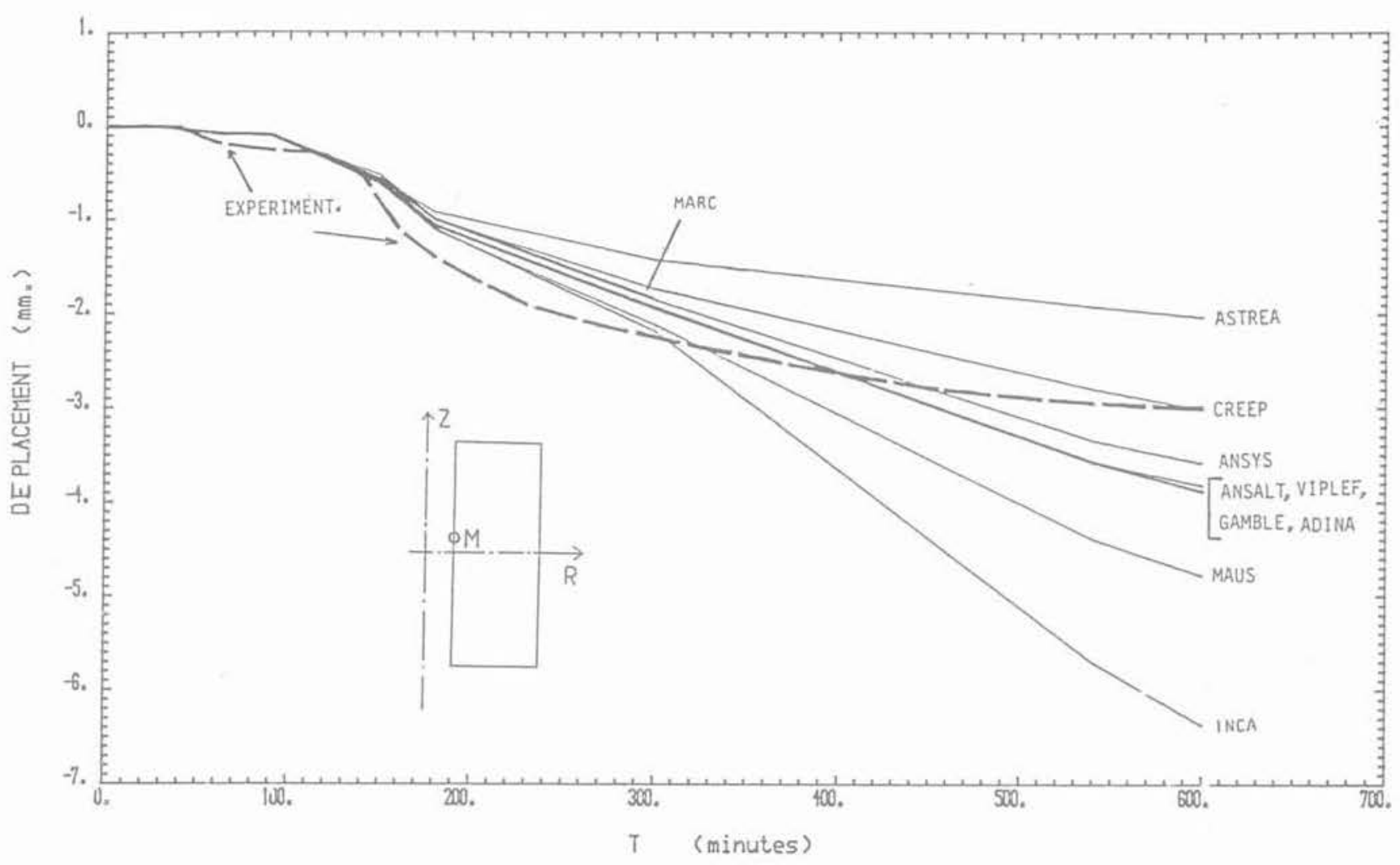

Fig. 5. - Niveau 2. Calcul mécanique. Cas de base.

Première comparaison entre convergence mesurée et convergences calculées à la paroi du forage.

Fig. 5. - Benchmark 2. Mechanical calculation. Base case.

First comparison between measured and calculated radial convergence of borehole wall.

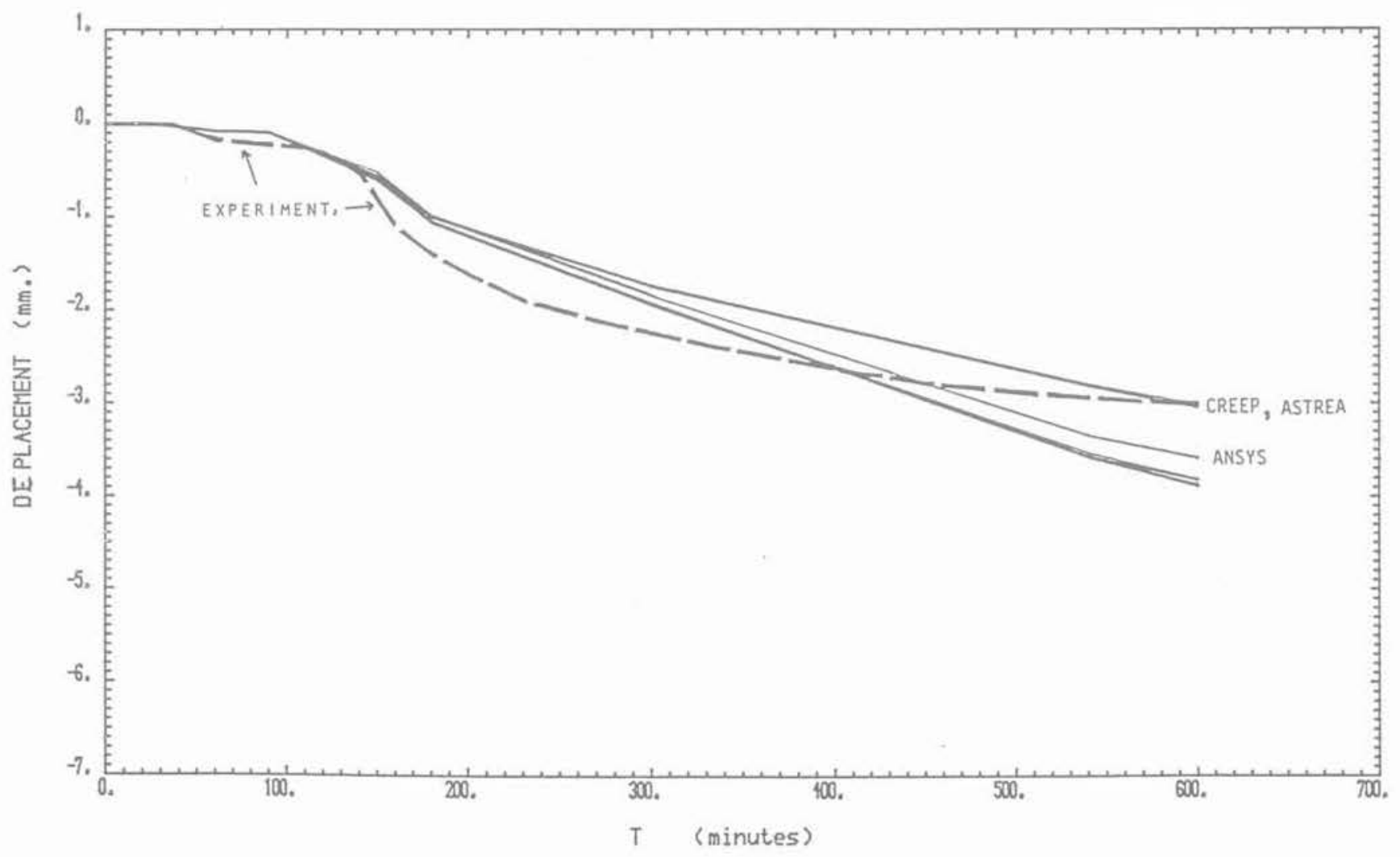

Fig. 6. - Niveau 2. Calcul mécanique. Cas de base.

Comparaison entre convergence mesurée et convergences calculées à la paroi du forage : résultats révisés.

Fig. 6. - Benchmark 2. Mechanical calculation. Base case.

Comparison between measured and calculated radial convergence of borehole wall: revised results. 
sée; on constate, sur la figure 6, le meilleur accord entre les prévisions numériques.

Il n'en reste pas moins clair que seul l'ordre de grandeur du résultat expérimental, et non pas sa a chronologie » exacte, avait pu être reproduit par les calculs, de manière néanmoins assez satisfaisante.

\subsection{Calcul mécanique. Variantes}

En vue d'améliorer l'accord entre les calculs et les mesures, un certain nombre de variantes ont été explorées, entre autres:

- calcul en déformations planes du milieu du forage;

- frottement parfait entre plateaux et sel

- autres équations de comportement pour le sel.

A titre d'exemple, on détaille ici une variante présentée par l'École des Mines (E.M.P.), concernant la rhéologie du sel, Sur base de quelques essais complémentai- res de fluage sur échantillons, un modèle de Lemaitre (LEMAITRE, 1970) avec écrouissage a pu être adapté au sel de Asse sous la forme :

$$
\dot{\varepsilon}_{c r}=\frac{\alpha \cdot\left[(\sigma / \mathrm{K})^{\beta} \cdot \exp (-\mathrm{A} / \mathrm{T})\right]^{1 / \alpha}}{\left(\varepsilon_{c t}\right)^{(1-\alpha) / \alpha}}
$$

avec : $\alpha=0,463 ; \beta=3,57 ; K=0,166$;

$\mathrm{A}=2540$.

valeurs obtenues expérimentalement par les tests de fluage, et $\mathrm{T}$ température absolue $\left({ }^{\circ} \mathrm{K}\right)$.

La figure 7 permet de comparer la courbe expérimentale de la convergence radiale et les deux "prévisions" par le code VIPLEF, respectivement pour le modèle * de base * et le modèle de Lemaître. Il est clair qu'une amélioration substantielle du résultat calculé est apportée par l'emploi d'un tel modèle rhéologique avec écrouissage.



Fig. 7. - Niveau 2. Calcul mécanique. Exemple de variante. Comparaison entre la convergence mesurée et les calculs par VIPLEF utilisant (a) le modèle "de base " et (b) le modèle de LEMAITTRE.

Fig. 7. - Benchmark 2. Mechanical calculation. Example of variant.

Comparison between measured borehole convergence and calculations with VIPLEF using (a) the base case model and (b) the LEMAITTRE model. 


\section{CONCLUSION ET PERSPECTIVES}

Les deux premiers «niveaux du projet COSA ont permis d'évaluer de façon satisfaisante les capacités actuelles des codes de calcul (et des équipes qui les exploitent) dans le domaine de la thermo-mécanique du sel, à l'échelle de la Communauté Européenne. Deux conclusions qualitatives émergent nettement du deuxième « niveau » :

- plus que les codes de calcul eux-mêmes, les modèles rhéologiques de comportement du sel doivent faire l'objet de recherches ultérieures:

- des problèmes de structures complexes, non linéaires et en régime transitoire, devraient de préférence être analysés par plusieurs équipes travaillant de manière indépendante si l'on veut obtenir des résuitats crédibles.

Le projet COSA se poursuit en 1987-1988 par un troisième niveau, avec les mêmes participants. Cette fois, il s'agit de recalculer un essal existant «in-situ», en vraie grandeur et de longue durée, dans le sel, analogue à une véritable situation d'évacuation de déchets émetteurs de chaleur. Une plus grande liberté sera accordée aux participants dans le choix de paramètres tels que les conditions de chargement, le type de modélisation géométrique; il sera intéressant de voir en quoi les résultats des calculs en seront affectés.

\section{BIBLIOGRAPHIE}

LEMAITRE, J. (1970), Sur la détermination des lois de comportement des matériaux élastovisco. plastiques. Publication ONERA n 135 , Paris.

LOWE, M.J.S., KNOWLES, N.C. (1986), The Community Project COSA : comparison of geo. mechanical computer codes for salt, Rapport C.C.E. $n^{\circ}$ EUR 10760, Luxembourg.

MORGAN, H.S., KRIEG, R.D., MATALUCCI, R.V. (1981), Computer Analysis of Nine Structural Codes used in the Second WIPP Benchmark Pro. blem. SANDIA Report SAND 81-1389, Sandia Laboratories, U.S.A.

ROEST, J.P.A., GRAMBERG, J. (1985), Acoustic Crosshole Measurements of Cataclastic Thermomechanical Behaviour of Rock Salt. Comptes rendus d'un séminaire conjoint C.C.E.-A.E.N. "Conception et instrumentation d'expériences in-situ en laboratoires souterrains ». Bruxelles, mai 1984 Balkema Publishers. (réf. C.C.E. n EUR 9575)

SIMON, R. (éditeur). (1985), Radioactive Waste Management and Disposal. Comptes rendus de la $2^{e}$ Conférence Communautaire, Luxembourg, avril 1985. Cambridge University Press (rêf. C.C.E. n० EUR 10163).

\section{REMERCIEMENTS}

L'auteur tient à remercier ici tous les participants au projet COSA pour leur efficace contribution au succès de cet exercice. 
